\title{
TYPES OF INVOLUTORIAL SPACE TRANSFORMATIONS ASSOCIATED WITH CERTAIN RATIONAL CURVES*
}

\author{
BY \\ AMOS HALE BLACK
}

\section{INTRODUCTION}

The purpose of this paper is to find and discuss the involutorial transformations belonging to the special complex of lines which meet a rational curve $r$ of order $m$, and having a pencil of invariant surfaces $\left|F_{n}\right|$ which contain the curve as an $(n-2)$-fold basis element. If $n=2$ the curve $r_{m}$ is not a basis curve. A pencil of quadrics and any rational curve always lead to a result contained among those found by Montesano. $\dagger$ The remaining admissible cases are as follows:

I. The pencils of surfaces of order $n, n \geqq 3$, which contain a straight line as an $(n-2)$-fold basis element. If $n=3$, in any plane containing the line there is a plane Cremona involution of order seven having for fundamental points four triple points which are not on the line and three double points which are on the line. The space involution is obtained by revolving the plane about the line. This case has already been treated. $f$

II. A pencil of cubic surfaces which contain simply (a) a conic, (b) a rational cubic, (c) a rational space quartic, (d) a rational space quintic.

III. A pencil of quartic surfaces which contain doubly (a) a conic, (b) a space cubic.

A pencil of cubic surfaces cannot contain as basis curve a rational curve of higher order than five, because it would necessarily contain all the quadrisecants. A pencil of quartic surfaces cannot contain doubly a basis curve of higher order than three, because it would necessarily contain all the trisecants. Similarly, pencils of surfaces of degree greater than four are inadmissible since they would necessarily contain all the bisecants.

In this paper we shall discuss the transformations defined in II and III. We shall, however, confine ourselves to the case where the residual intersection of any two surfaces of the pencil is not composite, except in $\mathrm{II}(\mathrm{d})$ and III(b) where the residual is necessarily composite.

\footnotetext{
* Presented to the Society, September 2, 1932; received by the editors May 15, 1932.

$\dagger$ Montesano, Su una classe di transformazioni razionali ed involutorie dello spazio de genere arbitrario $n$ e di grado $2 n+1$, Giornale di Mathematiche, vol. 31 (1892), pp. 36-50.

$\ddagger$ Miss E. T. Carroll, Systems of involutorial birational transformations contained multiply in special linear complexes, American Journal of Mathematics, vol. 54 (1932).
} 
It can be shown that III(a) can be transformed into II(a) by means of a quadratic involution.

The transformation III(b) is the most interesting of all the cases treated because it has a new kind of singularity. The other transformations have a finite number of parasitic lines, but the transformation III(b) has an infinite number of parasitic lines lying on a ruled surface in addition to ordinary parasitic lines.

Given a rational space curve $r_{m}$ defined by a homogeneous parameter $(\lambda, \mu)$ and a pencil of surfaces $\left|F_{n}\right|: r_{m}^{n-2}$. If we make the points of the curve and the surfaces of the pencil projective, then any point $P(y)$ in space will uniquely determine a surface $F_{n}$ of the pencil, a point $O(z)$ on $r_{m}$, and a line $P O$ of the complex of lines which meet $r_{m}$. The line $P O$ will cut $F_{n}$ in $P, O^{n-2}$ and a third point $P^{\prime}(x)$. We define $P^{\prime}(x)$ as the image of $P(y)$. Conversely, if we choose $P^{\prime}$ as the initial point we determine the same surface $F_{n}$, point $O$, and line of the complex. Hence $P$ is the image of $P^{\prime}$. The transformation is then involutorial and on a general line of the complex is one pair of points $P, P^{\prime}$ in involution.

Let the pencil of surfaces $\left|F_{n}\right|: r_{m}^{n-2}$ be

$$
\mu F\left(x_{1}, x_{2}, x_{3}, x_{4}\right)-\lambda F^{\prime}\left(x_{1}, x_{2}, x_{3}, x_{4}\right)=\mu F(x)-\lambda F^{\prime}(x)=0 .
$$

Call the residual base curve of the pencil $\gamma$. Since $r_{m}$ is rational the coördinates of any point $O(z)$ are

$$
x_{i}=z_{i}(\lambda, \mu) \quad(i=1,2,3,4)
$$

where $z_{i}(\lambda, \mu)$ is homogeneous and of degree $m$ in $(\lambda, \mu)$. Any point on the line joining $P(y)$ to $O(z)$ has coördinates

$$
x_{i}=\rho y_{i}+\sigma z_{i} \quad(i=1,2,3,4) .
$$

The value of $\rho / \sigma$ for $P^{\prime}(x)$ is given by

$$
\mu F(\rho y+\sigma z)-\lambda F^{\prime}(\rho y+\sigma z)=0 .
$$

Since $P$ is on (1) and $O$ is on (2) we find

$$
\rho\left[\mu F(z, y)-\lambda F^{\prime}(z, y)\right]+\sigma\left[\mu F(y, z)-\lambda F^{\prime}(y, z)\right]=0
$$

where $F(z, y), F^{\prime}(z, y)$ are the first polars of $F(y), F^{\prime}(y)$ with respect to $(z)$, and $F(y, z), F^{\prime}(y, z)$ are the first polars of $F(z), F^{\prime}(z)$ with respect to $(y)$. Hence $\rho / \sigma=-R / M$, where

$$
\begin{aligned}
R & =\mu F(y, z)-\lambda F^{\prime}(y, z), \\
M & =\mu F(z, y)-\lambda F^{\prime}(z, y) .
\end{aligned}
$$


The involutorial transformation is therefore expressed by

$$
x_{i}=y_{i} k-z_{i} M \quad(i=1,2,3,4)
$$

where $\lambda / \mu=F(y) / F^{\prime}(y)$.

If $M(y)=0, P$ and $P^{\prime}$ coincide, hence $M=0$ is the equation of the surface of invariant points.

At any point $O$ of $r_{m}$ each tangent plane of the associated surface $F_{n}$ intersects $F_{n}$ in a curve $C_{n}: O^{n-1}$. The whole $C_{n}$ is transformed into the point $O$. Conversely, the image of $O$ is $C_{n}$. As the point $O$ describes $r_{m}$ the $C_{n}$ generates the surface $R=0$.

With point $O$ on $r_{m}$ as vertex draw the cone $K$ of bisecants of $r_{m}$. On each generator of $K$ lies one point $P^{\prime}$, the image of $O$. Then the $F_{n}$ associated with $O$ and $K$ intersect in $r_{m}$ and a residual curve $C^{\prime}$ which is the part of the image of $r_{m}$ lying on $K$ and $F_{n}$. Since $r_{m}$ is rational, the equation of $K$ is homogeneous and of degree $(m-1)(m-2)$ in $(\lambda, \mu)$ and of degree $(m-1)$ in $(x)$. The image of $r_{m}$ lying on the bisecants of $r_{m}$ is a surface $R^{\prime}=0$ and is obtained by eliminating the parameters $(\lambda, \mu)$ between $K$ and $F_{n}$. Thus the total image of $r_{m}$ is $R+R^{\prime}$.

On each generator of the rational cone with vertex $P$ on $\gamma$ and standing on $r_{m}$ is one point $P^{\prime}$, the image of $P$. The locus of $P^{\prime}$ is a curve $C^{\prime \prime}$. As $P$ traces $\gamma$ the curve $C^{\prime \prime}$ generates a surface $\Gamma$ which is the total image of $\gamma$. Since any surface of the pencil (1) is invariant then the image of $F_{n}$ must contain $F_{n}$ and the images of $r_{m}$ and $\gamma$. Then the equation of $\Gamma$ is obtained by finding the image of $F_{n}$ :

$$
F_{n} \sim F_{n} R R^{\prime} \Gamma
$$

We shall consider II(c) in detail.

\section{CASE II (c)}

1. Equations of the transformation. We have a pencil of cubic surfaces $\left|F_{3}\right|: r_{4}$. The residual base curve $\gamma_{j}$ is of order five, genus one, and intersects $r_{4}$ in ten points. From (5) the equations of the transformation are

$$
I_{29}: x_{i}=y_{i} R_{28}-z_{i} M_{17}
$$$$
(i=1,2,3,4)
$$

where

$$
\begin{aligned}
& R_{28}=F^{\prime}(y) F(y, z)-F(y) F^{\prime}(y, z), \\
& M_{17}=F^{\prime}(y) F(z, y)-F(y) F^{\prime}(z, y) .
\end{aligned}
$$

$M_{17}=0$ is the equation of the surface of invariant points.

2. Images of the fundamental elements. The image of $O(z)$ on $r_{4}$ lying in the tangent plane of $F O$, the surface of the pencil associated with $O$, is a curve $\mathrm{C}_{3}: O^{2}$. As $O$ describes $r_{4}$ the $\mathrm{C}_{3}: O^{2}$ generates the surface $R_{28}$. In the direction 
of the two tangents of $C_{3}: O^{2}$ at $O$ the point $O$ is invariant. Hence two sheets of $R_{28}$ and $M_{17}$ have this plane for a common tangent plane. Thus two sheets of $R_{28}$ touch two sheets of $M_{17}$ along $r_{4}$. However, the point $O$ for these two sheets is a binode and counts for six in the intersection of $R_{28}$ and $M_{17}$.

Let $L$ be an arbitrary point on $r_{4}$.

The point $O$ has image $P^{\prime}$ on $O L$, the residual point of intersection of $O L$ and $F_{L}$. As $L$ describes $r_{4}, O L$ generates a cubic cone $K_{3}$, with one point $P^{\prime}$ on each generator. The locus of $P^{\prime}$ is then a curve of order $3+$ the number of times $O L$ is tangent to $F_{L}$ at $O$. Given $L$, the tangent plane at $O$ meets $r_{4}$ in two points $K_{1}, K_{2}$; given $K$, there is a unique point $L$. This $(1,2)$ correspondence on $r_{4}$ has three coincidences, and the locus of $P^{\prime}$ is $C_{6}: O^{3}, p=0$. As $O$ describes $r_{4}$ the $C_{6}$ generates a surface $R_{21}^{\prime}=0$.

The equation of $R_{21}^{\prime}$ may be obtained by eliminating the parameter between the cone $K_{3}(\lambda, \mu, x)=0$ and $F_{3}=\mu F(x)-\lambda F^{\prime}(x)=0$.

Each point $P^{\prime}$ of $C_{6}$ is perspective from $O$, hence $O$ is invariant in the three directions of the tangents to $C_{6}$ at $O$. Thus three sheets of $R_{21}^{\prime}$ are tangent respectively to three sheets of $M_{17}$ along $r_{4}$.

The tangent line to $r_{4}$ at $O$ lies on $K_{3}$ and also in the tangent plane to $F_{O}$. Hence $C_{3}: O^{2}$ and $C_{6}: O^{3}$ intersect in one point. As $O$ describes $r_{4}$ this point generates a curve $\delta_{11}$ which lies on both $R_{28}$ and $R_{21}{ }^{\prime}$.

The image of any point $P$ on $\gamma_{5}$ is a curve $C_{9}$ which lies on the quartic cone $K_{4}$ with vertex $P$ standing on $r_{4}$, cuts each generator in one point, and has five branches passing through $P$. Thus $P \sim C_{9}: P^{5}$. As $P$ traces $\gamma_{5}$ the $C_{9}$ generates a surface $\Gamma_{35}$ whose equation may be found by finding the image of any $F_{3}$ of the pencil:

$$
F_{3} \sim F_{3} R_{28} R_{21}{ }^{\prime} \Gamma_{35}
$$

The point $P$ is invariant in the directions of the five tangents of $C_{9}$ at $P$. Hence five sheets of $\Gamma_{35}$ are tangent respectively to five sheets of $M_{17}$ along $\gamma_{5}$.

3. Determination of the parasitic lines. On any $F_{3}$ lie twenty-seven lines. Whenever one of these lines passes through the associated point $O$ on $r_{4}$ it is parasitic. It is desired to know at how many points $O$ on $r_{4}$ a line on $F_{o}$ passes through $O$. To do this we map the cubic surface on the plane by means of cubic curves through six basis points $1,2,3,4,5,6$. In this plane a conic $C_{2}: 12 \sim r_{4}$ on $F_{3}$ and a curve of order seven $C_{7}: 1^{2} 2^{2} 3^{3} 4^{3} 5^{3} 6^{3} \sim \gamma_{5}$ on $F_{3}$. $\left[C_{2}, C_{7}\right]=10$ points. Therefore $\left[r_{4}, \gamma_{5}\right]=10$ points, as already indicated.

Consider any line $l$ on any $F_{3}$ of the pencil. This line meets any other surface $F_{3}^{\prime}$ of the pencil in three points, and since $F_{3}, F_{3}^{\prime}$ intersect in $r_{4}, \gamma_{5}$, only these three points must be on $r_{4}, \gamma_{5}$. Hence the lines $l$ may be classified as follows: 
$A$. Lines which meet $r_{4}$ three times, do not meet $\gamma_{5}$.

$B$. Lines meeting $r_{4}$ twice, meeting $\gamma_{5}$ once.

$C$. Lines meeting $r_{4}$ once, meeting $\gamma_{5}$ twice.

$D$. Lines which do not meet $r_{4}$, meet $\gamma_{5}$ three times.

We find

2 conics, conics containing 1 , or 2 , and $3,4,5,6$, meet $C_{2}$ in three points, do not meet $C_{7}$.

Hence there are two lines $A$ on $F_{3}$.

4 conics, conics containing 1,2 , and three of $3,4,5,6$, meet $C_{2}$ twice, meet $C_{7}$ once.

6 lines, joins of $3,4,5,6$ by pairs, meet $C_{2}$ twice, meet $C_{7}$ once.

Hence there are $4+6=10$ lines $B$ on $F_{3}$.

2 lines, images of 1,2 , meet $C_{2}$ once, meet $C_{7}$ twice.

8 lines, joins of 1 , or 2 , to 3 , or 4 , or 5 , or 6 , meet $C_{2}$ once, meet $C_{7}$ twice.

Hence there are $2+8=10$ lines $C$ on $F_{3}$.

1 line, join of 1,2 , does not meet $C_{2}$, meets $C_{7}$ three times.

4 lines, images of $3,4,5,6$, do not meet $C_{2}$, meet $C_{7}$ three times.

Hence there are $1+4=5$ lines $D$ on $F_{3}$.

In all there are $2+10+10+5=27$ lines on $F_{3}$.

The lines $D$ do not enter the problem since they do not meet $r_{4}$.

Given point $O$ on $r_{4}$. There are two lines $A$ on $F_{o}$. Each line meets $r_{4}$ three times, hence there are six points $K$. Conversely, given a point $K$, there is one line $A$, the trisecant of $r_{4}$ through $K$. This line will determine one surface of the pencil, hence one point $O$. Then there is a $(1,6)$ correspondence between the points $O$ and $K$. Since $r_{4}$ is rational there are $1+6=7$ coincidences. Hence there are seven parasitic lines which are trisecants of $r_{4}$ but do not meet $\gamma_{5}$. These lines are simple on $R_{28}, M_{17}$; double on $R_{21}^{\prime}$; do not lie on $\Gamma_{35}$.

Given point $O$ on $r_{4}$ there are ten lines $B$. Each line meets $r_{4}$ twice, hence twenty points $K$. Conversely, given a point $K$ there are five points $O$. To show this we construct the two cones with common vertex $K, K_{3}$ standing on $r_{4}$ and $K_{5}$ standing on $\gamma_{5}$. These cones intersect in fifteen lines of which ten are lines joining $K$ to the ten points common to $r_{4}, \gamma_{5}$, hence only five lines $B$. Each line will determine one point $O$. There is a $(5,20)$ correspondence between the points $O$ and $K$, hence $5+20=25$ coincidences. There are twentyfive parasitic lines which meet $r_{4}$ twice and meet $\gamma_{5}$ once. These lines are simple on $R_{28}, M_{17}, R_{21}{ }^{\prime}$, and $\Gamma_{35}$.

Given a point $O$ there are ten lines $C$. Each line meets $r_{4}$ once, hence ten points $K$. Conversely, given a point $K$, there are five lines $C$, the lines joining $K$ to the five apparent double points of $\Gamma_{5}$. Hence there is a $(5,10)$ correspond- 
ence between the points $O$ and $K$, and $5+10=15$ coincidences. There are fifteen parasitic lines which meet $r_{4}$ once and meet $\gamma_{5}$ twice. These lines are simple on $R_{28}, M_{17}$; double on $\Gamma_{35}$; do not lie on $R_{21}^{\prime}$.

In all there are forty-seven parasitic lines distributed as follows: All are simple on $R_{28}$ and $M_{17}$; seven are double on $R_{21}{ }^{\prime}$, do not lie on $\Gamma_{35}$; fifteen do not lie on $R_{21}^{\prime}$, are double on $\Gamma_{35}$; the remaining twenty-five are simple on both $R_{21}^{\prime}$ and $\Gamma_{35}$.

4. Table of images. We have the following table:

A general plane $a x_{1}+b x_{2}+c x_{3}+d x_{4} \sim S_{29}$. Since $S_{29}$ is linear in $R_{28}$ and $M_{17}$, the forty-seven parasitic lines are all simple on $S_{29}$. At each point $O$ of $r_{4}$ three sheets of $S_{29}$ have for common tangent plane the tangent plane of $F_{o}$.

$$
S_{1} \sim S_{29}: r_{+}^{9+3 t} \gamma_{5}^{9} 47 g
$$

where the $t$ in the multiplicity of $r_{4}$ means only that the multiplicity is due to contact.

$$
\begin{aligned}
& r_{4} \sim R_{28}: r_{4}^{9+2 t} \gamma_{5}^{9} 47 g+R_{21}^{\prime}: r_{4}^{7+2 t} \gamma_{5}^{6} 25 g 7 g^{2} \\
& \gamma_{5} \sim \Gamma_{35}: r_{4}^{10+5 t} \gamma_{5}^{11} 25 g 15 g^{2} \\
& M_{17}: r_{4}^{5+2 t} \gamma_{5}^{5} 47 g .
\end{aligned}
$$

The Jacobian is

$$
J_{112} \equiv R_{28}^{2} R_{21}^{\prime} \Gamma_{35}
$$

\section{CASE II(a)}

5. Equations of the transformation. Let $r_{2}$ be defined as the intersection of the quadric surface $H_{2}(x)=0$ and the plane $\pi$. The pencil (1) is $\left|F_{3}\right|: r_{2}$. The residual base curve is $\gamma_{7}$, genus five, meets $r_{2}$ in six points, and meets $\pi$ in a seventh point $Q$ not on $r_{2}$. From (5) the equations of the transformation are

$$
I_{17}: x_{i}=y_{i} R_{16}+z_{i} M_{11} \quad(i=1,2,3,4),
$$

where

$$
\begin{aligned}
R_{16} & =F^{\prime}(y) F(y, z)-F(y) F^{\prime}(y, z), \\
M_{11} & =F^{\prime}(y) F(z, y)-F(y) F^{\prime}(z, y) .
\end{aligned}
$$

$M_{11}=0$ is the equation of the surface of invariant points.

6. Images of the fundamental elements. The image of $O(z)$ lying in the tangent plane of $F_{O}$ is a curve $C_{3}: O^{2}$ which generates the surface $R_{16}$ as $O$ 
describes $r_{2}$. The point $O$ is invariant in the directions of the two tangents to $C_{3}$ at $O$, hence two sheets of $R_{16}$ and $M_{11}$ have a common tangent plane at all points of $r_{2}$.

The plane $\pi$ intersects the pencil $\left|F_{3}\right|$ in $r_{2}$ and a pencil of lines $|d|$ whose vertex is $Q$, and which is projective with the points of $r_{2}$. Let $L$ be an arbitrary point on $r_{2}$. The point $O$ has image $P^{\prime}$ on $O L$, the intersection of $O L$ and $d_{L}$, the line of $|d|$ associated with $L$. As $L$ describes $r_{2}, P^{\prime}$ generates a conic $C_{2}: O Q O_{1} O_{2} O_{3}$ where $O_{i}(i=1,2,3)$ are the points where $d_{L}$ passes through $L$. As $O$ describes $r_{2}, C_{2}$ generates the plane $\pi$. Thus the total image of $r_{2}$ is $R_{16}+\pi$.

The tangent to $r_{2}$ at $O$ is a line $O L, L=0$, and lies in the tangent plane of $F_{O}$ at $O$. Thus $C_{3}: O^{2}$ and $C_{2}: O Q O_{1} O_{2} O_{3}$ intersect in one point $P^{\prime}$. This point is the point of intersection of the tangent of $r_{2}$ at $O$ and $d_{o}$. As $O$ describes $r_{2}$ the point $P^{\prime}$ generates a curve $\delta_{3}$ which has a node at $Q$ and touches $r_{2}$ at the three points $O_{1}, O_{2}, O_{3}$. This is Lehmer's nodal cubic, ${ }^{*}$ and lies on $R_{16}$ and $\pi$.

The image of a point $P$ on $\gamma_{7}$ is a $C_{5}: P^{3}$. As $P$ describes $\gamma_{7}, C_{5}$ generates a surface $\Gamma_{31}$, whose equation is found from the image of any $F_{3} . F_{3} \sim F_{3} R_{16} \pi \Gamma_{31}$. Since $P$ is invariant in three directions, three sheets of $\Gamma_{31}$ are respectively tangent to three sheets of $M_{11}$ along $\gamma_{7}$.

Any plane passing through $Q$ will intersect $\gamma_{7}$ in six other points which lie on a conic. $\dagger$

7. Determination of the parasitic lines. We recall that all lines must meet the basis curves $r_{2}, \gamma_{7}$ in three points. In the plane $C_{2}: 1234 \sim r_{2}$ on $F_{3}$ and $C_{7}: 1^{22} 2^{2} 3^{2} 4^{25^{3}} 6^{3} \sim \gamma_{7}$ on $F_{3}$, hence $\left[C_{2}, C_{7}\right]=8$ points and $C_{7}$ is of genus five.

Now we find that there are three parasitic lines which meet $r_{2}$ twice and meet $\gamma_{7}$ once. These are the lines joining $Q$ respectively to $O_{1}, O_{2}, O_{3}$. They are simple on $R_{16}, \pi, M_{11}$, and $\Gamma_{31}$. There are twenty-six parasitic lines which meet $r_{2}$ once and meet $\gamma_{7}$ twice. These lines are simple on $R_{16}, M_{11}$; double on $\Gamma_{31}$; do not lie on $\pi$.

8. Table of images. We have the following table:

$$
\begin{gathered}
S_{1} \sim S_{17}: r_{2}^{5+3 t} \gamma_{7}^{5} 29 g \\
r_{2} \sim R_{16}: r_{2}^{5+2 t} \gamma_{7}^{5} 29 g+\pi: r_{2} 3 g \\
\gamma_{7} \sim \Gamma_{31}: r_{2}^{8+7 t} \gamma_{7}^{9} 3 g 26 g^{2} \\
M_{11}: r_{2}^{3+2 t} \gamma_{7}^{3} 29 g .
\end{gathered}
$$

The Jacobian is $J_{64} \equiv R_{16}{ }^{2} \pi \Gamma_{31}$.

* D. N. Lehmer, Constructive theory of the unicursal cubic by synthetic methods, these Transactions, vol. 3 (1902), pp. 372-376.

† R. Sturm, Synthetische Untersuchungen über Flächen dritter Ordnung, Leipzig, 1867, p. 229. 


\section{CAse II(b)}

9. Equations of the transformation. Let $r_{3}$ be a space cubic curve. The pencil (1) is now $\left|F_{3}\right|: r_{3}$. The residual base curve is $\gamma_{6}, p=3$, and intersects $r_{3}$ in eight points. From (5) the equations of the transformation are

$$
I_{23}: x_{i}=y_{i} R_{22}-z_{i} M_{14} \quad(i=1,2,3,4),
$$

where

$$
\begin{gathered}
R_{22}=F^{\prime}(y) F(y, z)-F(y) F^{\prime}(y, z), \\
M_{14}=F^{\prime}(y) F(z, y)-F(y) F^{\prime}(z, y) .
\end{gathered}
$$

$M_{14}=0$ is the equation of the surface of invariant points.

The image of $r_{3}$ lying in the tangent planes of $F_{O}$ is $R_{22}$. Again 'wo sheets of $R_{22}$ and $M_{14}$ are tangent along $r_{3}$.

The image of a point $O$ on $r_{3}$ which lies on the bisecants of $r_{3}$ is a $C_{4}: O^{2}$. As $O$ describes $r_{3}$ the $C_{4}$ generates a surface $R_{8}^{\prime}=0$. Two sheets of $R_{8}^{\prime}$ are respectively tangent to two sheets of $M_{14}$ along $r_{3}$.

The locus of the point common to $C_{3}: O^{2}$ and $C_{4}: O^{2}$ is a curve $\delta_{7}$ lying on $R_{22}$ and $R_{8}^{\prime}$.

The image of a point $P$ on $\gamma_{6}$ is a $C_{7}: P^{4}$. As $P$ traces $\gamma_{6}$ the $C_{7}$ generates a surface $\Gamma_{36}$. Four sheets of $\Gamma_{36}$ are respectively tangent to four sheets of $M_{14}$ along $\gamma_{6}$.

10. Determination of the parasitic lines. In the plane $C_{2}: 123 \sim r_{3}$ on $F_{3}$ and $C_{7}: 1^{2} 2^{2} 3^{2} 4^{3} 5^{3} 6^{3}(p=3) \sim \gamma_{6}$ on $F_{3}$. Hence $\left[C_{2}, C_{7}\right]=8$ points.

We find that there are thirty-eight parasitic lines distributed as follows: All are simple on $R_{22}$ and $M_{14}$; sixteen are simple on both $R_{8}^{\prime}$ and $\Gamma_{36}$; twentytwo do not lie on $R_{8}{ }^{\prime}$, are double on $\Gamma_{36}$.

11. Table of images. We have the following table:

$$
\begin{aligned}
& S_{1} \sim S_{23}: r_{3}^{7+3 t} \gamma_{6}^{7} 38 g \\
& r_{3} \sim R_{22}: r_{3}^{7+2 t} \gamma_{6}^{7} 38 g+R_{8}^{\prime}: r_{3}^{3+1 t} \gamma_{6}^{8} 16 g \\
& \gamma_{6} \sim \Gamma_{36}: r_{3}^{10+6 t} \gamma_{6}^{11} 16 g 22 g^{2} \\
& M_{14}: r_{3}^{4+2 t} \gamma_{6}^{4} 38 g .
\end{aligned}
$$

The Jacobian is $J_{88} \equiv R_{22}{ }^{2} R_{8}^{\prime} \Gamma_{36}$. 


\section{CASE II $\left(b^{\prime}\right)$}

12. If $r_{3}$ is a rational plane cubic then the plane $\pi$ of $r_{3}$ factors out of the transformation and (10) reduces to

$$
I_{22}: x_{i}=y_{i} R_{21}-z_{i} M_{13} \quad(i=1,2,3,4)
$$

where $R_{21}, M_{13}$ are given by (11) after factoring out $\pi . M_{13}=0$ is the equation of the surface of invariant points. $R_{21}=0$ is the total image of $r_{3}$. The image of $\gamma_{6}$ is a surface $\Gamma_{42}$. There are only thirty-three parasitic lines and they are all simple on $R_{21}, M_{13}$; and double on $\Gamma_{42}$.

$$
\begin{aligned}
& S_{1} \sim S_{22}: r_{3}^{6+3 t} \gamma_{6}^{7} 33 g \\
& r_{3} \sim R_{21}: r_{3}^{6+2 t} \gamma_{6}^{7} 33 g \\
& \gamma_{6} \sim \Gamma_{42}: r_{3}^{12+6 t} \gamma_{6}^{133} 3 g^{2} ; \\
& M_{13}: r_{3}^{3+2 t} \gamma_{6}^{4} 33 g .
\end{aligned}
$$

The Jacobian is $J_{84} \equiv R_{21}{ }^{2} \Gamma_{42}$.

\section{CASE II(d)}

13. Equations of the transformation. The pencil (1) becomes $\left|F_{3}\right|: r_{5}$. The curve $r_{5}$ has one quadrisecant $l$, hence this line lies on every surface of the pencil. The residual base curve is a space cubic $\gamma_{3}$ which meets $r_{5}$ in eight points, but does not meet $l$. From (5) the equations of the transformation are

$$
I_{35}: x_{i}=y_{i} R_{34}-z_{i} M_{20} \quad(i=1,2,3,4),
$$

where

$$
\begin{aligned}
R_{35} & =F^{\prime}(y) F(y, z)-F(y) F^{\prime}(y, z), \\
M_{20} & =F^{\prime}(y) F(z, y)-F(y) F^{\prime}(z, y) .
\end{aligned}
$$

Again $M_{20}=0$ is the equation of the surface of invariant points.

14. Images of fundamental elements. The image of a point $O$ on $r_{5}$ lying in the tangent plane of $F_{O}$ at $O$ is a $C_{3}: O^{2}$. As $O$ describes $r_{5}$ the $C_{3}$ generates the surface $R_{34}$. Two sheets of $R_{34}$ and two sheets of $M_{20}$ have a common tangent plane along $r_{5}$.

The image of a point $O$ lying on the bisecants of $r_{5}$ is a $C_{8}: O^{4}$. As $O$ describes $r_{5}$ the $C_{8}$ generates a surface $R_{40}$. Four sheets of $R_{40}{ }^{\prime}$ are respectively tangent to four sheets of $M_{20}$ along $r_{5}$. The total image of $r_{5}$ is $R_{34}+R_{40}{ }^{\prime}$.

The tangent line to $r_{5}$ at $O$ cuts $C_{3}: O^{2}$ and $C_{8}: O^{4}$ in a common point. As $O$ describes $r_{5}$ this point generates a curve $\delta_{15}$ which lies on both $R_{34}$ and $R_{40}{ }^{\prime}$. The image of a point $Q$ on $l$ is a $C_{7}: Q^{2}$. As $Q$ describes $l$, the $C_{7}$ generates a 
surface $L_{4}$. Two sheets of $L_{4}$ are tangent respectively to two sheets of $M_{20}$ along $l$. The equation of $L_{4}$ is found as follows: The plane through $l$ and $O$ intersects $F_{o}$ in $l$ and a residual conic $C_{2}$ which is the part of the image of $l$ which lies on $F_{O}$. We obtain the equation of $L_{4}$ by eliminating the parameter $(\lambda, \mu)$ between the equations of the plane and $F_{O}$.

The image of a point $P$ on $\gamma_{3}$ is a curve $C_{11}: P^{6}$. As $P$ describes $\gamma_{3}$ the $C_{11}$ generates a surface $\Gamma_{24}$. Six sheets of $\Gamma_{24}$ are tangent respectively to six sheets of $M_{20}$ along $\gamma_{3}$. The equation of $\Gamma_{24}$ is found from the image of any $F_{3}$. Thus: $F_{3} \sim F_{3} R_{34} R_{40}^{\prime} L_{4} \Gamma_{24}$.

15. Determination of the parasitic lines. In the plane a conic $C_{2}: 1 \sim r_{5}$ $(p=0)$ on $F_{3}$; a conic $C_{2}^{\prime}: 23456 \sim l$ on $F_{3}$; a quintic $C_{5}: 1^{2} 2^{2} 3^{2} 4^{2} 5^{2} 6^{2} \sim \gamma_{3}$ $(p=0)$ on $F_{3}$. Hence $\left[C_{2}, C_{2}^{\prime}\right]=4$ points, $\left[C_{2}, C_{5}\right]=8$ points, $\left[C_{2}^{\prime}, C_{5}\right]=0$ points.

In the present problem seven types of lines enter:

$A$. Lines which meet $r_{5}$ three times, do not meet $l$ or $\gamma_{3}$.

$B$. Lines which meet $r_{5}$ twice, do not meet $l$, meet $\gamma_{3}$ once.

$C$. Lines which meet $r_{5}$ twice, meet $l$ once, do not meet $\gamma_{3}$.

$D$. Lines which meet $r_{5}$ once, do not meet $l$, meet $\gamma_{3}$ twice.

$E$. Lines which meet $r_{5}$ once, meet $l$ once, meet $\gamma_{3}$ once.

$F$. Lines which do not meet $r_{5}$, meet $l$ once, meet $\gamma_{3}$ twice.

$G$. The line $l$ itself which meets $r_{5}$ four times, does not meet $\gamma_{3}$.

There are no lines $C$, and lines $F$ do not enter the problem. From the map, and then the number of coincidences on $r_{5}$ we find the following: There are eighteen parasitic lines of type $A$, twenty-four of type $B$, two of type $D$, eight of type $E$.

The map fails to give the number of times $l$ is counted as a parasitic line. We shall determine this in another manner. Denote by $Q_{i}(i=1,2,3,4)$ the four points common to $l$ and $r_{5}$. Now $l$ lies in the tangent plane of $F_{Q}$ at $Q_{i}$, hence is parasitic. But $l$ appears as a parasitic line at each of the four points $Q_{i}$ independently of the other three. Hence $l$ counts as a parasitic line four times. We shall think of it as four parasitic lines. These lines are simple on $R_{34}$ and $M_{20}$; triple on $R_{40}{ }^{\prime}$; do not lie on $L_{4}$ or $\Gamma_{24}$.

Thus there are fifty-six parasitic lines distributed as follows: All are simple on $R_{34}, M_{20}$; eighteen are double on $R_{40}{ }^{\prime}$, do not lie on $L_{4}, \Gamma_{24}$; twenty-four are simple on $R_{40}{ }^{\prime}, \Gamma_{24}$, but do not lie on $L_{4}$; two are double on $\Gamma_{24}$ but do not lie on $L_{4}, R_{40}{ }^{\prime}$; eight are simple on $L_{4}, \Gamma_{24}$ but do not lie on $R_{40}{ }^{\prime}$; four are triple on $R_{40}{ }^{\prime}$ but do not lie on $L_{4}, \Gamma_{24}$. 
16. Table of images. We have the following table:

$$
\begin{aligned}
S_{1} & \sim S_{35}: r_{5}^{11+3 t} \gamma_{3}^{11} l^{11} 56 g \\
r_{5} & \sim R_{34}: r_{5}^{11+2 t} \gamma_{3}^{11} l^{11} 56 g+R_{40}^{\prime}: r_{5}^{13+3 t} \gamma_{3}^{12} l^{12} 24 g 18 g^{2} 4 g^{3} \\
\gamma_{3} & \sim \Gamma_{24}: r_{5}^{7+3 t} \gamma_{3}^{8} l^{7} 24 g 8 g 2 g^{2} ; \\
l & \sim L_{4}: r_{5}^{1+1 t} \gamma_{3} l^{2} 8 g \\
M_{20} & : r_{5}^{6+2 t} \gamma_{3}^{6} l^{6} 56 g
\end{aligned}
$$

The Jacobian is $J_{136} \equiv R_{34}{ }^{2} R_{40}^{\prime} L_{4} \Gamma_{24}$.

\section{CAse III(a)}

17. The pencil (1) is now $\left|F_{4}\right|: r_{2}{ }^{2}$. The residual base curve is a $\gamma_{8}$ which intersects $r_{2}$ in eight points. If we transform $\left|F_{4}\right|$ by a quadratic involution whose fundamental elements are the conic $r_{2}$ and a point on $\gamma_{8}$, it transforms into $\left|F_{3}\right|: r_{2}$, or Case II(a).

$$
\text { CASE III(b) }
$$

18. Equations of the transformation. Given the equations of the space cubic curve $r_{3}$ as

$$
x_{1} / x_{2}=x_{2} / x_{3}=x_{3} / x_{4}=\lambda / \mu,
$$

and let

$$
F(x)=0, \quad F^{\prime}(x)=0
$$

be two quartic surfaces which contain $r_{3}$ as a double basis curve. They intersect in $r_{3}$ and a residual composite quartic curve which consists of four straight lines $l_{i}$, each of which is a bisecant of $r_{3}$. Then

$$
\left|F_{4}\right|: r_{3}^{2} l_{1} l_{2} l_{3} l_{4}
$$

A surface of the pencil $\mu F(x)-\lambda F^{\prime}(x)$ through the point $P(y)$ determines $\lambda / \mu=F(y) / F^{\prime}(y)$. The coördinates of a point on the line joining $F(y)$ to $O(z) \equiv\left(F^{3}, F^{2} F^{\prime}, F F^{\prime 2}, F^{\prime 3}\right)$ are given by

$$
x_{i}=\rho y_{i}+\sigma z_{i} \quad(i=1,2,3,4) .
$$

The residual point of intersection of $P O$ with $F_{4}(x)$ after making reductions is given by

$$
\rho \Delta_{1}(z, y)+\sigma \Delta_{2}(z, y)=0
$$

where $\Delta_{i}(z, y)$ is the $i$ th polar of $F_{4}(y)$ with respect to $(z)$. 
$\Delta_{1}(z, y)$ and $\Delta_{2}(z, y)$ are homogeneous and respectively of degree nineteen and thirty in $(y)$. However, $\Delta_{1}$ and $\Delta_{2}$ have a common factor $R_{10}^{\prime}$ which is of the tenth degree in $(y)$. Hence

$$
\Delta_{1}(y) \equiv M_{9}(y) R_{10}^{\prime}(y), \quad \Delta_{2}(y) \equiv R_{20}(y) R_{10}^{\prime}(y) .
$$

The equations of the transformation are now

$$
I_{21}: x_{i}=y_{i} R_{20}-z_{i} M_{9} \quad(i=1,2,3,4) .
$$

$M_{9}=0$ is the equation of the surface of invariant points.

We shall find that although $R_{10}{ }^{\prime}$ factors out of the transformation it still plays the most important rôle of any surface in the transformation.

19. Images of the fundamental elements. Given any point $P$ on $l_{i}$. Any point $O$ on $r_{3}$ will determine an associated $F_{4}$ and the line $O P$ will cut $F_{4}$ in $O^{2}$, $P$, and a third point $P^{\prime}$ which is the image of $P$. As $O$ generates $r_{3}$ the point $P^{\prime}$ generates a curve $C_{5}$ which lies on the cubic cone $K_{3}$ standing on $r_{3}$ with vertex $P$, cuts each generator in one point $P^{\prime}$, and has two branches passing through $P$. Then $P \sim C_{5}: P^{2}$. As $P$ describes $l_{i}$ the $C_{5}: P^{2}$ generates a surface of order five which is the total image of $l_{i}$.

We shall determine the equation of this surface in an alternate manner. Suppose $l_{i}$ is the intersection of two planes $u_{i}(x)=0, v_{i}(x)=0$. The pencil of planes

$$
\mu u_{i}(x)-\lambda v_{i}(x)=0
$$

is projective with the pencil $\left|F_{4}(x)\right|$. Any point $O$ on $r_{3}$ will determine a surface $F_{4}(x)$ and a plane of (20) passing through $O$. The plane will cut $F_{4}(x)$ in $l_{i}$ and a cubic curve $C_{3}$ which is the part of the image of $l_{i}$ lying on this $F_{4}(x)$. Thus the whole image of $l_{i}$ can be obtained by eliminating the parameter $(\lambda, \mu)$ between the pencils $(20)$ and $\left|F_{4}(x)\right|$. Thus

$$
L_{5, i} \equiv F(x) v_{i}(x)-F^{\prime}(x) u_{i}(x)=0 .
$$

There are four such surfaces $L_{5, i}$.

The two tangent planes to the associated $F_{4}$ at $O$ on $r_{3}$ cut the $F_{4}$ in two quartic curves, each having a triple point at $O, 2 C_{4}: O^{3}$. As $O$ traces $r_{3}$ the $2 C_{4}: O^{3}$ generate the surface $\Delta_{2}(z, y)$.

Any $F_{4}(x): r_{3}{ }^{2}$ is ruled and through each point on $r_{3}$ pass two generators $g, g^{\prime}$ of $F_{4}$. One generator lies in each of the tangent planes of $F_{4}$ at $O$. Thus both the quartic curves are composite and consist of a cubic and a generator of $F_{4}$,

$$
2 C_{4} \equiv C_{3} g+C_{3}^{\prime} g^{\prime}
$$

The two cubic curves generate the surface $R_{20}$, while the two generators, $g, g^{\prime}$, generate the surface $R_{10}^{\prime}$. 
20. The surface $R_{10}{ }^{\prime}=0$. The cone $K_{2}$ standing on $r_{3}$ with vertex at a point $O$ on $r_{3}$ and the $F_{4}$ associated with $O$ intersect in $r_{3}$ and two lines, the two generators $g, g^{\prime}$ of $F_{4}$ passing through $O$. The locus of $g, g^{\prime}$ is $R_{10}{ }^{\prime}$.

From (15) we find three independent quadrics passing through $r_{3}$ to be

$$
H_{1}(x)=x_{1} x_{3}-x_{2}^{2}=0, H_{2}(x)=x_{2} x_{4}-x_{3}^{2}=0, H_{3}(x)=x_{1} x_{4}-x_{2} x_{3}=0,
$$

and the equation of $K_{2}$, vertex $O(\lambda, \mu)$, is

$$
\mu^{2} H_{1}(x)+\lambda^{2} H_{2}(x)-\lambda \mu H_{3}(x)=0,
$$

hence

$$
R_{10}^{\prime} \equiv F^{\prime 2}(x) H_{1}(x)+F^{2}(x) H_{2}(x)-F(x) F^{\prime}(x) H_{3}(x)=0 .
$$

The generators $g, g^{\prime}$ are parasitic lines. Then through each point of $r_{3}$ pass two parasitic lines whose locus is $R_{10}{ }^{\prime}=0$, a ruled surface. It has five sheets passing through $r_{3}$. The two sheets of $R_{10}{ }^{\prime}$ determined by the two generators $g, g^{\prime}$ of the $F_{4}$ associated with the point we shall call the "at" sheets. Now $g$ and $g^{\prime}$ are bisecants of $r_{3}$ hence intersect $r_{3}$ in two other points $O_{1}, O_{2}$. At $O_{1}$ the associated $F_{4}$ has two generators $g_{1}, g_{1}^{\prime}$, which determine the "at" sheets of $R_{10}{ }^{\prime}$ through $O_{1}$. The line $g$ is a generator of $R_{10}{ }^{\prime}$ but does not lie on the $F_{4}$ associated with $O_{1}$. Its associated point is $O$. We shall think of it as coming from point $O$. Through $O_{1}$ pass three such lines $g$ whose origin is at some other point. The three sheets of $R_{10}{ }^{\prime}$ determined by these generators we shall call the "from" sheets.

21. Determination of the parasitic lines. In general neither $g$ nor $g^{\prime}$ lies on any of the other surfaces of the transformation. We wish to find which of these lines do lie on other surfaces, and any other parasitic lines which may arise.

There are four points on $r_{3}$ at which the $g$ and $g^{\prime}$ of the associated $F_{4}$ coincide and thus are contact generators of $R_{10}{ }^{\prime}$, and also lie on $S_{21}, R_{20}$ and $M_{9}$.

At three points of $r_{3}$ the associated $F_{4}$ is composite, consisting of two quadric surfaces each of which contains $r_{3}$. Two generators of each quadric pass through the point. Hence there are four parasitic lines which pass through each of the three points. Two are generators of $R_{10}{ }^{\prime}$ but the other two are not, as they are not bisecants of $r_{3}$. All of the generators of $r_{10}^{\prime}$ are bisecants of $r_{3}$, hence there are six parasitic lines which do not lie on $R_{10}{ }^{\prime}$. They are distributed as follows: All are simple on $S_{21}, R_{20}, M_{9}$; three lie on each of the surfaces $L_{5, i}$, such that just one is common to $L_{5, j}, L_{5, i}(i \neq j)$.

22. Table of images. A general plane $S_{1} \sim S_{21}$ having nine sheets passing through $r_{3}$ such that each of the tangent planes of the associated $F_{4}$ is the 
common tangent plane of three sheets at all points of $r_{3}$. The three remaining sheets are tangent to the three "from" sheets of $R_{10}{ }^{\prime}$. The image of $r_{3}$ for the latter contact is $R_{10}{ }^{\prime}$. There are five sheets passing, through each $l_{i}$. Six parasitic lines are simple and four double on $S_{21}$.

$$
S_{1} \sim S_{21}: r_{3}^{9+3 t+3 t+3 t^{\prime}} 4 l_{i}^{5} 6 g 4 g^{2} .
$$

The surface $R_{20}$ has nine sheets passing through $r_{3}$ such that each of the tangent planes of the associated $F_{4}$ is the common tangent plane of two sheets at all points of $r_{\mathbf{s}}$. Three other sheets are tangent to the three "from" sheets of $R_{10}{ }^{\prime}$. There are five sheets passing through each $l_{i}$. All ten parasitic lines are simple on $R_{\mathbf{2 0}}$.

$$
R_{20}: r_{3}^{9+2 t+2 t+3 t^{\prime}} 4 l_{i}^{5} 6 g 4 g .
$$

The surface of invariant points $M_{9}$ has four sheets passing through $r_{3}$ such that each tangent plane of the associated $F_{4}$ is the common tangent plane of two sheets at all points of $r_{3}$. There are two sheets passing through each $l_{i}$. Six parasitic lines are simple and four double on $M_{9}$.

$$
M_{9}: r_{3}^{4+2 t+2 t} 4 l_{i}^{2} 6 g 4 g^{2} .
$$

Any surface $L_{5, i}$ has two sheets passing through $r_{3}$ such that the tangent planes of these sheets are the tangent planes of the associated $F_{4}$ at all points of $r_{3}$. There are two sheets passing through $l_{i}$. These two sheets are tangent to the two sheets of $M_{9}$ through $l_{i}$. These are the sheets determined by the two tangents of $C_{5}: P^{2}$ at $P$. There is just one sheet passing through each of the three remaining lines $l_{j}$. There are three simple parasitic lines lying on $L_{5, i}$ distributed respectively on the three $L_{5, j}$.

$$
L_{5, i}: r_{3}^{2+1 t+1 t}{ }_{i}^{2} 3 l_{j} 3 g .
$$

The tangent planes of the two "at" sheets of $R_{10}$ ' are the tangent planes of the associated $F_{4}$ at all points of $r_{3}$. The three "from" sheets are tangent to three sheets of $S_{21}$ and $R_{20}$ at all points of $r_{3}$. There are two sheets passing through each $l_{i}$. Four parasitic lines are double on $R_{10}{ }^{\prime}$.

$$
R_{10}^{\prime}: r_{3}^{5+1 t+1 t} 4 l_{i}^{2} 4 g^{2} .
$$

Collecting,

$$
\begin{aligned}
& S_{1} \sim S_{21}: r_{3}^{9+3 t+3 t+3 t^{\prime}} 4 l_{i}^{5} 6 g 4 g^{2} \\
& r_{3} \sim R_{20}: r_{3}^{9+2 t+2 t+3 t^{\prime}} 4 l_{i}^{5} 6 g 4 g+R_{10}^{\prime}: r_{3}^{5+1 t+1 t} 4 l_{i}^{2} 4 g^{2}
\end{aligned}
$$




$$
\begin{aligned}
l_{i} & \sim L_{5, i}: r_{3}^{2+1 t+1 t} l_{i}^{2} 3 l 3 g \\
M_{9} & \sim M_{9}: r_{3}^{4+1 t+1 t} 4 l_{i}^{2} 6 g 4 g^{2} \\
S_{21} & \sim\left(R_{20} R_{10}^{\prime}\right){ }^{9} R_{20}^{3} R_{10}^{\prime} 4 L_{5, i}^{2} S_{1} ; \\
R_{20} & \sim\left(R_{20} R_{10}^{\prime}\right)^{9} R_{20}^{2} R_{10}^{\prime} 4 L_{5, i}^{5} \\
M_{9} & \sim\left(R_{20} R_{10}^{\prime}\right)^{4} R_{20} 4 L_{5, i}^{2} M_{9} ; \\
L_{5, i} & \sim\left(R_{20} R_{10}^{\prime}\right)^{2} R_{20} L_{5, i}^{2} 3 L_{5, i} ; \\
R_{10}^{\prime} & \sim\left(R_{20} R_{10}^{\prime}\right)^{5} R_{20} 4 L_{5, i}^{2}
\end{aligned}
$$

The Jacobian is $J_{80} \equiv R_{20}^{2} R_{10}^{\prime 2} L_{5,1} L_{5,2} L_{5,3} L_{5,4}$.

23. Generalization. In the preceding cases there has always been a $(1,1)$ correspondence between the points of $r_{m}$ and the surfaces of $\left|F_{n}\right|$. Let us assume the correspondence is $(1, k)$. The case where $r_{m}$ is a straight line has been treated by Miss E. T. Carroll (loc. cit.). Then a general point $P(y)$ will determine just one point $O(z)$ hence one point $P^{\prime}(x)$, but given $O(z)$ there are $k$ associated surfaces $F_{n}$. A general line of the complex through $O$ will cut each surface in a pair of points $P, P^{\prime}$. Hence on each line of the complex are $k$ pairs of points in involution. We shall illustrate by working II(c) in detail.

24. Equations of the transformation. The coördinates of a point $O(z)$ on $r_{4}$ are given by (2)

$$
x_{i}=z_{i}(\lambda, \mu) \quad(i=1,2,3,4),
$$

but the pencil of surfaces $\left|F_{3}\right|: r_{4}$ is written

$$
m F(x)-l F^{\prime}(x)=0,
$$

where $\mu \phi_{1}(l, m)-\lambda \phi_{2}(l, m)=0$, the $\phi_{i}(l, m)$ being homogeneous forms of degree $k$ in $(l, m)$. Proceeding exactly as before the equations of the transformation are found to be

$$
I_{24 k+5}: x_{i}=y_{i} R_{24 k+4}-z_{i} M_{12 k+5} \quad(i=1,2,3,4),
$$

where

$$
R_{24 k+4}=m F(y, z)-l F^{\prime}(y, z), M_{12 k+5}=m F(z, y)-l F^{\prime}(z, y),
$$

and $l / m=F(y) / F^{\prime}(y), \lambda / \mu=\phi_{1}(l, m) / \phi_{2}(l, m) . M_{12 k+5}=0$ is the equation of the surface of invariant points.

25. Images of the fundamental elements. Associated with a point $O$ on $r_{4}$ are $k$ surfaces $F_{3}$. In the tangent plane of each $F_{3}$ at $O$ lies a $C_{3}: O^{2}$ which is the image of $O$. Thus $O \sim k C_{3}: O^{2}$. As $O$ describes $r_{4}$ the $k C_{3}: O^{2}$ generate the surface $R_{24 k+4}$. The point $O$ is invariant in two directions in each tangent plane, 
hence each of the $k$ tangent planes is the common tangent plane of two sheets of $R_{24 k+4}$ and $M_{12 k+5}$ along $r_{4}$.

The image of $O$ which lies on the bisecants of $r_{4}$ is a $C_{5 k+1}: O^{2 k+1}$ which lies on the cubic cone $K_{3}$ with vertex $O$ standing on $r_{4}$, cuts each generator in $k$ points and has $2 k+1$ branches through $O$. As $O$ describes $r_{4}$ the $C_{5 k+1}: O^{2 k+1}$ generates a surface $R_{18 k+3}^{\prime}$. There are $2 k+1$ sheets of $R_{18 k+3}^{\prime}$ which are tangent respectively to $2 k+1$ sheets of $M_{12 k+5}$ along $r_{4}$.

The locus of the $k$ points common to $C_{5 k+1}$ and $k C_{3}$ is a curve $\delta_{10 k+1}$ which lies on both $R_{24 k+4}$ and $R_{18 k+3}^{\prime}$.

The image of a point $P$ on $\gamma_{5}$ is a $C_{8 k+1}: P^{4 k+1}$ which lies on the quartic cone $K_{4}$ with vertex $P$ standing on $r_{4}$. As $P$ describes $\gamma_{5}$ the $C_{8 k+1}$ generates a surface $\Gamma_{30 k+5}$. There are $4 k+1$ sheets of $\Gamma_{30 k+5}$ which are tangent respectively to $4 k+1$ sheets of $M_{12 k+5}$ along $\gamma_{5}$.

26. Determination of the parasitic lines. The map of the cubic surface on a plane is the same as in $\S 3$, hence on any $F_{3}$ are two lines $A$, ten lines $B$, and ten lines $C$. However, the number of coincidences is different.

Given point $O$ on $r_{4}$ there are two lines $A$ on each of the $k$ associated surfaces $F_{3}$. Each line meets $r_{4}$ in three points, hence $6 k$ points $K$. Conversely, given a point $K$ there is one line $A$, the trisecant of $r_{4}$ through $K$. This line determines one point $O$. There is a $(1,6 k)$ correspondence between the points $O$ and $K$, hence $1+6 k$ coincidences. There are $1+6 k$ parasitic lines of type $A$ which are simple on $S_{24 k+5}, R_{24 k+4}, M_{12 k+5}$; double on $R_{18 k+3}^{\prime}$; do not lie on $\Gamma_{30 k+5}$.

Similarly there are $5+20 k$ parasitic lines of type $B$ which are simple on all the surfaces $S_{24 k+5}, R_{24 k+4}, M_{12 k+5}, R_{18 k+3}^{\prime}, \Gamma_{30 k+5}$; and $5+10 k$ parasitic lines of type $C$ which are simple on $S_{24 k+5}, R_{24 k+4}, M_{12 k+5}$, double on $\Gamma_{30 k+5}$, do not lie on $R_{18 k+3}^{\prime}$.

27. Table of images. We have the following table:

$$
\begin{aligned}
& S_{1} \sim S_{24 k+5}: r_{4}^{8 k+1+3 t} \gamma_{5}^{8 k+1}(11+36 k) g \\
& r_{4} \sim R_{24 k+4}: r_{4}^{8 k+1+2 t} \gamma_{5}^{8 k+1}(11+36 k) g \\
& \quad \quad \quad R_{18 k+3}^{\prime}: r_{4}^{6 k+1+2 t} \gamma_{5}^{6 k}(5+20 k) g(1+6 k) g^{2} \\
& \gamma_{5} \sim \Gamma_{30 k+5}: r_{4}^{10 k+5 t} \gamma_{5}^{10 k+1}(5+20 k) g(5+10 k) g^{2} ; \\
& M_{12 k+5}: r_{4}^{4 k+1 \div 2 t} \gamma_{5}^{4 k+1}(11+36 k) g .
\end{aligned}
$$

The Jacobian is $J_{4}(24 k+4) \equiv R_{24 k+4}^{2} R_{18 k+3}^{\prime} \Gamma_{30 k+5}$.

Cornell University, ITHACA, N. Y. 